\title{
From Hierarchical BIP to Petri Calculus ${ }^{\star}$
}

\author{
Roberto Bruni ${ }^{1}$, Hernán Melgratti ${ }^{2}$, and Ugo Montanari ${ }^{1}$ \\ 1 Dipartimento di Informatica, Università di Pisa, Italy \\ 2 Departamento de Computación, FCEyN, \\ Universidad de Buenos Aires - CONICET, Argentina
}

\begin{abstract}
We focus on Hierarchical BIP, an extension of Joseph Sifakis et al's BIP component framework, to provide a semantics-preserving, compositional encoding in the Petri calculus, a recently proposed algebra of stateless connectors and one-position buffers.
\end{abstract}

\section{Introduction}

In recent years Joseph Sifakis has successfully pursued a research strand focussed on a component framework called BIP [2], that has also been implemented in a language and a tool-set. BIP is a component framework for constructing systems by superposing three layers of modelling:

1) Behaviour, the lowest level, representing the sequential computation of individual components as automata whose arcs are labelled by sets of ports. The sets of ports of any two different components are disjoint, i.e., each port is uniquely assigned to a component.

2) Interaction, the second level, defining the allowed interactions between components. An interaction is just a set of ports typically of different components.

3) Priority, the top layer, assigning priorities to interactions to enforce scheduling policies, typically with the aim of reducing the size of the state space.

In the absence of priorities, the interaction layer of BIP admits the algebraic presentation given in 3 and comparisons with other models have been shown in 7/111, see 8, for an overview. In particular, an equivalent version of BIP systems is presented in [7] in terms of a compositional encoding in Petri nets with boundaries 11 .

Here we investigate hierarchically structured BIP systems and show that previous correspondence results on ordinay BIP can be extended to deal with Hierarchical BIP (HBIP) as defined in 10. HBIP systems are possibly formed by the combination of other HBIP systems, each seen as an ordinary component whose ports are its interactions. We exploit the Petri calculus, a calculus of stateful connectors introduced in 11, to encode in a compositional way HBIP systems while flattening them. Notably the encoding of components and of interactions can be given separately in the Petri calculus and then assembled by ordinary Petri calculus composition.

* Research supported by European FET-IST-257414 Integrated Project ASCENS, Progetto MIUR PRIN CINA Prot. 2010LHT4KM, ANPCyT Project BID-PICT2008-00319.

S. Bensalem et al. (Eds.): FPS 2014 (Sifakis Festschrift), LNCS 8415, pp. 54-68, 2014.

(C) Springer-Verlag Berlin Heidelberg 2014 
Structure of the paper. In $\S 2$ we recall the main background on BIP and on the Petri calculus to keep the paper self-contained. In $\S 3$ we define Hierarchical BIP and in $\S 4$ we present the main result of the paper, namely the compositional encoding from $\mathrm{HBI}(\mathrm{P})$ to the Petri calculus. In $\S[5$ we give some concluding remarks and discuss alternative approaches to HBIP. A toy running example of a client-server system is used to illustrate the main notions and constructions.

\section{Background}

\subsection{The BIP Component Framework}

This section reports on the formal definition of BIP as presented in [4. Since we disregard priorities, we call $\mathrm{BI}(\mathrm{P})$ the framework presented here.

Definition 1 (Interaction). Given a set of ports $P$, an interaction over $P$ is a non-empty subset $a \subseteq P$.

We write an interaction $\left\{p_{1}, p_{2}, \ldots, p_{n}\right\}$ as $p_{1} p_{2} \ldots p_{n}$ and $a \downarrow_{P_{i}}$ for the projection of an interaction $a \subseteq P$ over the set of ports $P_{i} \subseteq P$, i.e., $a \downarrow_{P_{i}}=a \cap P_{i}$. Projection extends to sets of interactions in the following way $\gamma_{\downarrow_{P}}=\left\{a \downarrow_{P} \mid a \in\right.$ $\left.\gamma \wedge a \downarrow_{P} \neq \varnothing\right\}$.

Definition 2 (Component). A component $B=(Q, P, \rightarrow)$ is a transition system where $Q$ is a set of states, $P$ is a set of ports, and $\rightarrow \subseteq Q \times 2^{P} \times Q$ is the set of labelled transitions.

As usual, we write $q \stackrel{a}{\rightarrow} q^{\prime}$ to denote the transition $\left(q, a, q^{\prime}\right) \in \rightarrow$. An interaction $a$ is enabled in $q$, denoted $q \stackrel{a}{\rightarrow}$, iff there exists $q^{\prime}$ s.t. $q \stackrel{a}{\rightarrow} q^{\prime}$. By abusing the notation, we will also write $q \stackrel{\varnothing}{\rightarrow} q$ for any $q$.

Definition $3\left(\mathbf{B I}(\mathbf{P})\right.$ system). $A \mathrm{BI}(\mathrm{P})$ system $B=\gamma\left(B_{1}, \ldots, B_{n}\right)$ is the composition of a finite set $\left\{B_{i}\right\}_{i=1}^{n}$ of components $B_{i}=\left(Q_{i}, P_{i}, \rightarrow_{i}\right)$ such that their sets of ports are pairwise disjoint, i.e., $P_{i} \cap P_{j}=\varnothing$ for $i \neq j$ parameterized by a set $\gamma \subseteq 2^{P}$ of interactions over the set of ports $P=\biguplus_{i=1}^{n} P_{i}$.

The semantics of a BIP system $\gamma\left(B_{1}, \ldots, B_{n}\right)$ is given by the transition system $\left(Q, P, \rightarrow_{\gamma}\right)$, with $Q=\Pi_{i} Q_{i}, P=\biguplus_{i=1}^{n} P_{i}$ and $\rightarrow_{\gamma} \subseteq Q \times 2^{P} \times Q$ is the least set of transitions satisfying the following inference rule

$$
\frac{a \in \gamma \quad \forall i \in 1 . . n: q_{i} \stackrel{a \downarrow_{P_{i}}}{\longrightarrow} q_{i}^{\prime}}{\left(q_{1}, \ldots, q_{n}\right) \stackrel{a}{\rightarrow} \gamma\left(q_{1}^{\prime}, \ldots, q_{n}^{\prime}\right)}
$$

Example 1. Consider the $\mathrm{BI}(\mathrm{P})$ system shown in Fig. 1, which contains a component Server that sequentially interacts with a component Client. The Server accepts a request from the Client thanks to the interaction $\{$ acpt, req $\}$. Then, the server can successfully answer the request by returning a value to the client (interaction $\{r e t, r e s p\}$ ) or can fail the request by rising an error signal, which is handled by the client (interaction $\{$ err, hdle $\}$ ). 


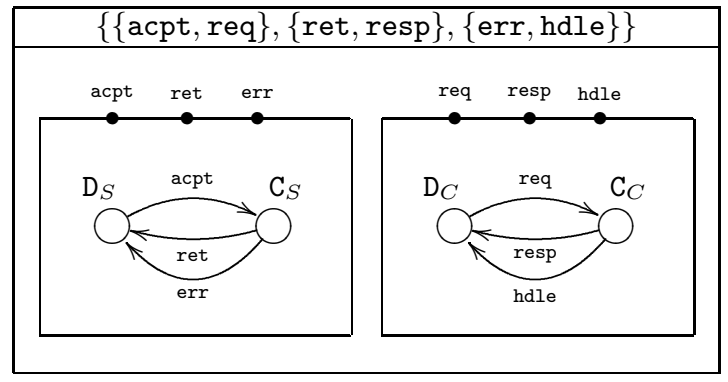

Fig. 1. A simple client/server $\mathrm{BI}(\mathrm{P})$ system

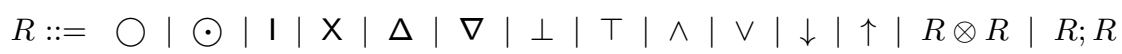

Fig. 2. Petri calculus grammar

\subsection{Petri Calculus}

The Petri calculus [1] enriches the algebra of stateless connectors from [5] with one-place buffers along [116].

Terms of the Petri Calculus are defined by the grammar in Fig. 2, It consists of the following constants plus parallel and sequential composition: the empty place $\bigcirc$, the full place $\odot$, the identity wire I, the twist (also swap, or symmetry) $X$, the duplicator (also sync) $\Delta$ and its dual $\nabla$, the mutex (also choice) $\wedge$ and its dual $\vee$, the hiding (also bang) $\perp$ and its dual $T$, the inaction $\downarrow$ and its dual $\uparrow$. The diagrammatical representation of terms is shown in Fig. 3 . For $n \in \mathbb{N}$, we write $\underline{n}$ to denote the finite ordinal $\underline{n} \stackrel{\text { def }}{=}\{0,1, \ldots, n-1\}$.

Any term has a unique associated sort (also called type) $(k, l)$ with $k, l \in \mathbb{N}$, that fixes the size $k$ of the left (input) interface and the size $l$ of the right (output) interface of $P$. The type of constants are as follows: $\bigcirc, \odot$, and I have type $(1,1)$, $X:(2,2), \Delta$ and $\wedge$ have type $(1,2)$ and their duals $\nabla$ and $\vee$ have type $(2,1)$, $\perp$ and $\downarrow$ have type $(1,0)$ and their duals $\top$ and $\uparrow$ have type $(0,1)$. The sort inference rules for composed processes are in Fig. 4 .

The operational semantics is defined by the rules in Fig. 5 , where $x, y \in\{0,1\}$. The labels $\alpha, \beta, \rho, \sigma$ of transitions are binary strings, all transitions are sortpreserving, and if $R \underset{\beta}{\stackrel{\alpha}{\longrightarrow}} R^{\prime}$ with $R, R^{\prime}:(n, m)$, then $\# \alpha=n$ and $\# \beta=m$. Notably, bisimilarity induced by such a transition system is a congruence.

Due to space limitation we omit details here and refer the interested reader to 9 .

Compound Terms. For the translation presented in $\S 4$, we shall need additional families of compound terms, indexed by $n \in \mathbb{N}_{+}$and $k \in \mathbb{N}$ (duals are omitted): 


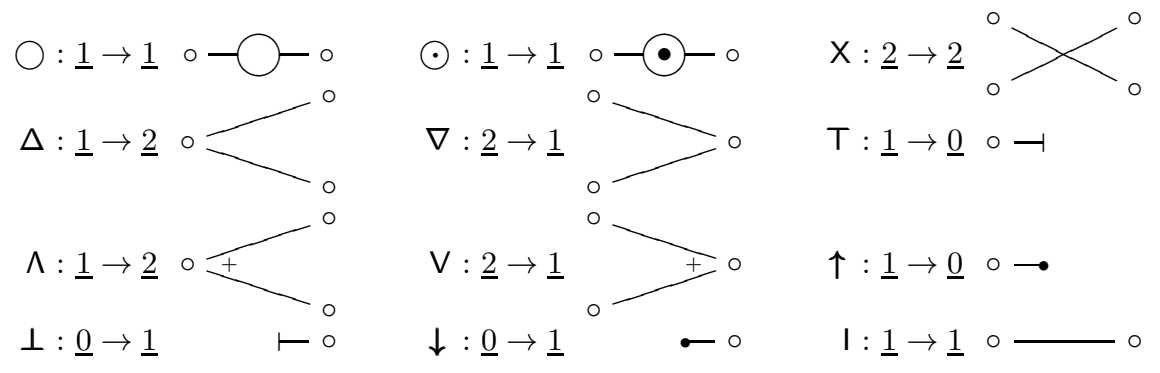

Fig. 3. Graphical representation of terms

$$
\frac{R:(k, l) \quad R^{\prime}:(m, n)}{R \otimes R^{\prime}:(k+m, l+n)} \quad \frac{R:(k, n) \quad R^{\prime}:(n, l)}{R ; R^{\prime}:(k, l)}
$$

Fig. 4. Sort inference rules

$$
\begin{array}{cccccc}
\mathrm{I}_{n}:(n, n) & \mathrm{T}_{n}:(0, n) & \uparrow_{n}:(0, n) & \mathrm{X}_{n}:(n+1, n+1) \\
\Delta_{n}:(n, 2 n) & \Lambda_{n}:(n, 2 n) & \Delta_{n}^{k}:(n, k * n) & \Lambda_{n}^{k}:(n, k * n) & d_{n}:(0,2 n)
\end{array}
$$

Intuitively, $\mathrm{I}_{n}, \mathrm{~T}_{n}$ and $\uparrow{ }_{n}$ correspond to $n$ parallel copies of I, $\mathrm{T}$ and $\uparrow$, respectively. Connector $\Delta_{n}$ (and its dual $\nabla_{n}$ ) is similar to $\Delta$ but duplicates $n$ wires in the other interface, while $\Delta_{n}^{k}$ (and its dual $\nabla_{n}$ ) replicates $k$-times the $n$ wires of the other interface. Connector $d_{n}$ (and its dual $e_{n}$ ) stands for the synchronisation of $n$ pairs of wires. We now give the definitions:

$$
\begin{aligned}
& \mathrm{I}_{n}=\otimes_{n} \mathrm{I} \quad \mathrm{X}_{1}=\mathrm{X} \quad \mathrm{X}_{n+1}=\left(\mathrm{X}_{n} \otimes \mathrm{I}\right) ;\left(\mathrm{I}_{n} \otimes \mathrm{X}\right) \\
& \uparrow_{n}=\bigotimes_{n} \uparrow \quad \Delta_{1}=\Delta \quad \Delta_{n+1}=\left(\Delta \otimes \Delta_{n}\right) ;\left(\mathrm{I} \otimes \mathrm{X}_{n} \otimes \mathrm{I}_{n}\right) \\
& \mathrm{T}_{n}=\otimes_{n} \mathrm{\top} \quad \Lambda_{1}=\Lambda \quad \Lambda_{n+1}=\left(\Lambda \otimes \Lambda_{n}\right) ;\left(\mathrm{I} \otimes \mathrm{X}_{n} \otimes \mathrm{I}_{n}\right) \\
& d_{n}=\mathrm{T}_{n} ; \Delta_{n} \quad \Delta_{n}^{0}=\mathrm{T}_{n} \quad \Delta_{n}^{k+1}=\Delta_{n} ;\left(\Delta_{n}^{k} \otimes \mathrm{I}_{n}\right) \\
& \Lambda_{n}^{0}=\uparrow_{n} \quad \Lambda_{n}^{k+1}=\Lambda_{n} ;\left(\Lambda_{n}^{k} \otimes \mathrm{I}_{n}\right)
\end{aligned}
$$

The behaviour of compound terms is characterised by the next proposition.

Proposition 1 (from [6]). For $n>0$,

1. $\mathrm{X}_{n} \underset{\beta}{\stackrel{\alpha}{\rightarrow}} t$ iff $t=\mathrm{X}_{n}, \alpha=h_{0} \ldots h_{n}$ and $\beta=h_{1} \ldots h_{n} h_{0}$.

2. $\Delta_{n} \underset{\beta}{\stackrel{\alpha}{\rightarrow}}$ t iff $t=\Delta_{n}, \# \alpha=n, \# \beta=2 n$ and $\alpha_{i}=\beta_{i}=\beta_{n+i}$ for all $i<n$.

3. $\nabla_{n} \underset{\beta}{\stackrel{\alpha}{\rightarrow}}$ t iff $t=\nabla_{n}, \# \alpha=2 n, \# \beta=n$ and $\alpha_{i}=\alpha_{n+i}=\beta_{i}$ for all $i<n$.

4. $\Lambda_{n} \underset{\beta}{\stackrel{\alpha}{\rightarrow}} t$ iff $t=\Lambda_{n}, \# \alpha=n, \# \beta=2 n, \alpha_{i}=\beta_{i}+\beta_{n+i}$ for all $i<n$.

5. $\mathrm{V}_{n} \underset{\beta}{\stackrel{\alpha}{\rightarrow}}$ t iff $t=\mathrm{V}_{n}, \# \alpha=2 n, \# \beta=n, \beta_{i}=\alpha_{i}+\alpha_{n+i}$ for all $i<n$. 


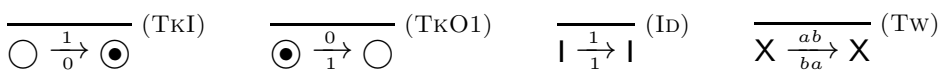

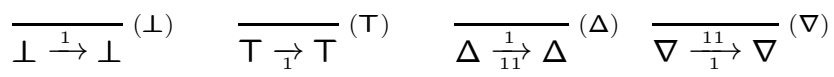

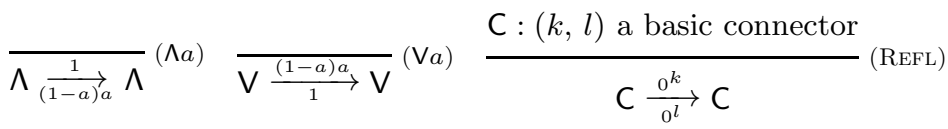

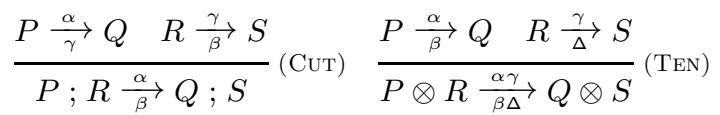

Fig. 5. Operational semantics for the Petri Calculus

6. $\Lambda_{l}^{n} \underset{\beta}{\stackrel{\alpha}{\rightarrow}}$ t iff $t=\Lambda_{l}^{n}, \# \alpha=l, \# \beta=n l$ and $\alpha_{i}=\Sigma_{j<n} \beta_{j l+i}$ for all $i<l$.

7. $\mathrm{V}_{l}^{n} \underset{\beta}{\stackrel{\alpha}{\rightarrow}}$ t iff $t=\mathrm{V}_{l}^{n}, \# \alpha=n l, \# \beta=l$ and $\beta_{i}=\Sigma_{j<n} \alpha_{j l+i}$ for all $i<l$.

8. $\Delta_{l}^{n} \underset{\beta}{\stackrel{\alpha}{\rightarrow}}$ tiff $t=\Delta_{l}^{n}, \# \alpha=l, \# \beta=n l$ and $\beta_{l j+i}=\alpha_{i}$ for all $i<l, j<n$.

9. $\nabla_{l}^{n} \underset{\beta}{\stackrel{\alpha}{\rightarrow}} t$ iff $t=\nabla_{l}^{n}, \# \alpha=n l, \# \beta=l$ and $\beta_{i}=\alpha_{l j+i}$ for all $i<l$ and $j<n$.

10. $d_{n} \underset{\beta}{\stackrel{\alpha}{\rightarrow}}$ t iff $t=d_{n}, \# \alpha=0, \# \beta=2 n$ and $\beta_{i}=\beta_{n+i}$ for all $i<n$ and $j<n$.

Relational Forms The encoding proposed in $\S 4$ uses two classes of terms of the Petri calculus, called the left and right relational forms, that represent functions as Petri calculus terms [1].

For any $h \in \mathbb{N}$, there is a bijection $\ulcorner-\urcorner: 2^{\underline{h}} \rightarrow\{0,1\}^{h}$ with

$$
\ulcorner U\urcorner_{i} \stackrel{\text { def }}{=}\left\{\begin{array}{l}
1 \text { if } i \in U \\
0 \text { otherwise }
\end{array}\right.
$$

For $\Theta$ a set of Petri calculus terms, let $T_{\Theta}$ denote the set of terms generated by the following grammar:

$$
T_{\Theta}::=\theta \in \Theta||\left|T_{\Theta} \otimes T_{\Theta}\right| T_{\Theta} ; T_{\Theta} .
$$

We shall use $t_{\Theta}$ to range over terms of $T_{\Theta}$.

Definition 4. A term $t:(k, l)$ is in right relational form when it is in

$$
T_{\{\perp\}} ; T_{\{\Delta\}} ; T_{\{\mathrm{X}\}} ; T_{\{\mathrm{V}\}} ; T_{\{\uparrow\}} .
$$

Dually, $t$ is said to be in left relational form when it is in

$$
T_{\{\downarrow\}} ; T_{\{\Lambda\}} ; T_{\{\mathrm{X}\}} ; T_{\{\nabla\}} ; T_{\{\mathrm{T}\}} \cdot
$$

The following result spells out the significance of the relational forms. 


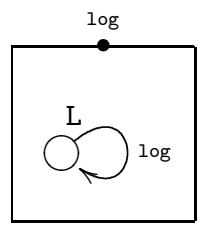

Fig. 6. Component Logging

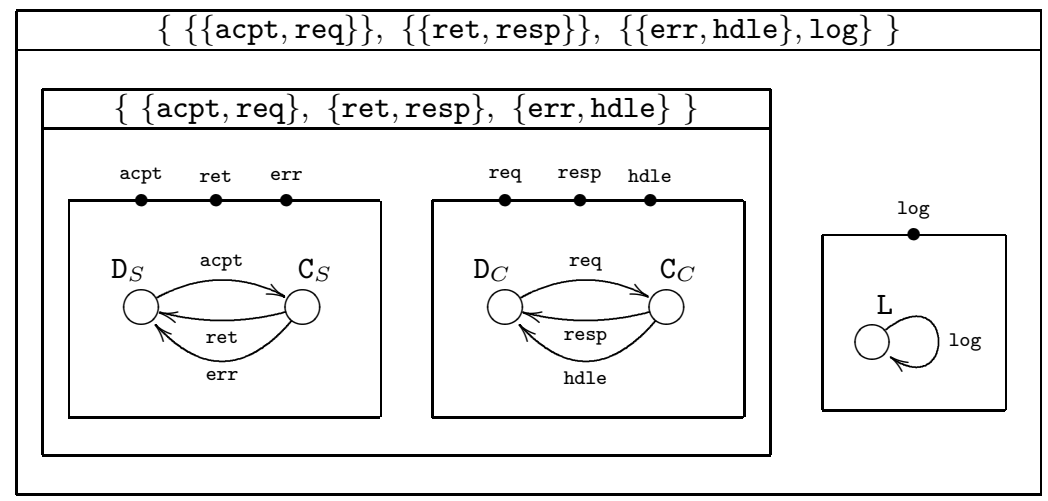

Fig. 7. A simple $\mathrm{BI}(\mathrm{P})$ system

Lemma 1 (From [11]). For each function $f: \underline{k} \rightarrow 2^{\underline{l}}$ there exists a term $\rho_{f}:$ $(k, l)$ in right relational form, the dynamics of which are characterised by the following:

$$
\begin{array}{r}
\rho_{f} \stackrel{\alpha}{\beta} \rho_{f} \Leftrightarrow \exists U \subseteq \underline{k} \text { s.t. } \forall u, v \in U . u \neq v \Rightarrow f(u) \cap f(v)=\varnothing, \alpha=\ulcorner U\urcorner \\
\text { and } \beta=\ulcorner f(U)\urcorner
\end{array}
$$

The symmetric result holds for functions $f: \underline{k} \rightarrow 2^{\underline{l}}$ and terms $t:(l, k)$ in left relational form. That is, there exists $\lambda_{f}:(l, k)$ in left relational form with semantics

$$
\begin{array}{r}
\lambda_{f} \underset{\beta}{\stackrel{\alpha}{\beta}} \lambda_{f} \Leftrightarrow \exists U \subseteq \underline{k} \text { s.t. } \forall u, v \in U . u \neq v \Rightarrow f(u) \cap f(v)=\varnothing, \beta=\ulcorner U\urcorner \\
\text { and } \alpha=\ulcorner f(U)\urcorner
\end{array}
$$

\section{Hierarchical BIP Systems}

In this section we address the hierarchical composition of $\mathrm{BI}(\mathrm{P})$ systems, i.e., $\mathrm{BI}(\mathrm{P})$ systems can be taken as components of larger systems.

Example 2. Consider the scenario introduced in Example 1] which should be extended with a logging functionality in order to record all error responses sent by the component Server. Assume we already have the simple component for logging depicted in Fig. 6. In this case, we would like to consider the system in 
Fig. 1 as a single component to define a new $\mathrm{BI}(\mathrm{P})$ system, as the one shown in Fig. 7. We remark that the interface (i.e., the set of ports) exposed by the client/server subsystem is just its set of interactions. This ensures that the composed system does not change the behaviour of the underlying subsystems.

Next definition formally introduces the notion of hierarchical composition of systems

Definition 5 (HBI(P) system). A Hierarchical $B I(P)$ system $(H B I(P))$ is either

- a $B I(P)$ component $B=(Q, P, \rightarrow)$ with interface $\iota(B)=P$; or

- a composite system $B=\gamma\left(B_{1}, \ldots, B_{n}\right)$ with interface $\iota(B)=\gamma$ where $\left\{B_{1}, \ldots, B_{n}\right\}$ is a set of hierarchical $B I(P)$ systems with pairwise disjoint interfaces, i.e., $\iota\left(B_{i}\right) \cap \iota\left(B_{j}\right)=\varnothing$ for $i \neq j$, and $\gamma$ is a set of interactions over $\uplus_{i=1}^{n} \iota\left(B_{i}\right)$.

The semantics of $\mathrm{HBI}(\mathrm{P})$ systems is defined analogously to that of $\mathrm{BI}(\mathrm{P})$ systems as the synchronous execution of the transitions of its constituent components matching one defined interaction. We start by defining the state space $\mathbb{Q}_{B}$ of a $\operatorname{HBI}(\mathrm{P})$ system $B$ as follows:

$-\mathbb{Q}_{B}=Q$ if $B=(Q, P, \rightarrow)$

$-\mathbb{Q}_{B}=\mathbb{Q}_{B_{1}} \times \ldots \times \mathbb{Q}_{B_{n}}$ if $B=\gamma\left(B_{1}, \ldots, B_{n}\right)$.

Then, the semantics of a composite $\operatorname{HBI}(\mathrm{P})$ system $B$ is given by the transition system $\left(\mathbb{Q}_{B}, \iota(B), \rightarrow\right)$ where $\rightarrow \subseteq \mathbb{Q}_{B} \times \iota(B) \times \mathbb{Q}_{B}$ is the least set of transitions satisfying the following inference rules

$$
\frac{a \in \iota(B) \quad \forall i \in 1 . . n: q_{i} \stackrel{a \downarrow_{\iota\left(B_{i}\right)}}{\longrightarrow} q_{i}^{\prime}}{\left(q_{1}, \ldots, q_{n}\right) \stackrel{a}{\rightarrow}\left(q_{1}^{\prime}, \ldots, q_{n}^{\prime}\right)}
$$

\section{HBI(P) Systems as Petri Calculus Terms}

This section gives an encoding of $\mathrm{HBI}(\mathrm{P})$ systems into the Petri calculus. Note that, differently from $\mathrm{BI}(\mathrm{P})$, the Petri calculus uses consecutive natural numbers to designate ports over interfaces. In order to establish a correspondence between $\mathrm{HBI}(\mathrm{P})$ systems and Petri calculus terms, we will map names into natural numbers, i.e., given a finite set $S$ with $k=\# S$, we use $w_{S}$ to denote an injective function $w_{S}: S \rightarrow \underline{k}$ that orders the elements of $S$. By abusing the notation, we write $w_{S}$ to also denote its expected extension $w_{S}: 2^{S} \rightarrow 2^{\underline{k}}$.

\subsection{Encoding of Basic Components}

We first address the encoding of basic components (Definition 2) as Petri calculus terms. The encoding of components follows along the lines of the encoding of 


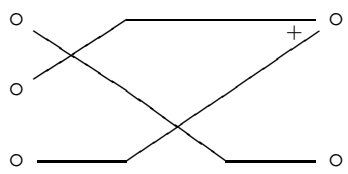

(a) $\rho_{\text {target } \rightarrow}$

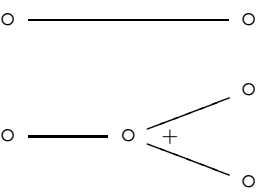

(b) $\lambda_{\text {source } \rightarrow}$

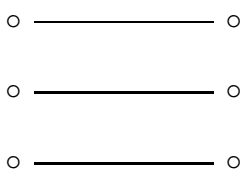

(c) $\rho_{l b l} \rightarrow$

Fig. 8. Petri calculus terms for relational forms

Petri nets proposed in [9, although it is simpler due to the fact that components are just sequential systems.

Given a component $B=(Q, P, \rightarrow)$ with $s$ transitions (i.e., $\# \rightarrow=s)$, we rely on the functions $w_{Q}, w_{P}$ and $w_{\rightarrow}$ that respectively order the elements in $Q, P$ and $\rightarrow$. In addition, we will use the following three functions source $_{\rightarrow}$, target $_{\rightarrow}$, $l b l_{\rightarrow}$, which map a labelled transition belonging to $\rightarrow$ into its source, target and labels, when considering the sets of names just as ordinals.

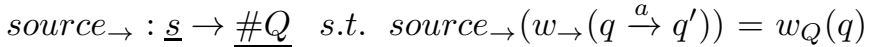

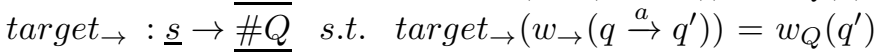

$$
\begin{aligned}
& l b l_{\rightarrow} \quad: \underline{s} \rightarrow \underline{\overline{2^{\# P}}} \text { s.t. } \quad l b l_{\rightarrow}\left(w_{\rightarrow}\left(q \stackrel{a}{\rightarrow} q^{\prime}\right)\right)=w_{P}(a)
\end{aligned}
$$

Thanks to Lemma 1, we know that the relational forms $\lambda_{\text {source }_{\rightarrow}, \rho_{\text {target }} \rightarrow}$ and $\rho_{l b l} \rightarrow$ exist and their behaviours are in tight correspondence with the associated functions.

Example 3. Consider the component Server in Fig. 1 and assume the following ordering functions

$$
\begin{array}{lll}
w_{Q}\left(\mathrm{D}_{S}\right)=0 & w_{P}(\text { acpt })=0 & w_{\rightarrow}\left(\mathrm{D}_{S} \stackrel{\text { acpt }}{\longrightarrow} \mathrm{C}_{S}\right)=0 \\
w_{Q}\left(\mathrm{C}_{S}\right)=1 & w_{P}(\text { ret })=1 & w_{\rightarrow}\left(\mathrm{C}_{S} \stackrel{\text { ret }}{\longrightarrow} \mathrm{D}_{S}\right)=1 \\
& w_{P}(\text { err })=2 & w_{\rightarrow}\left(\mathrm{C}_{S} \stackrel{\text { err }}{\longrightarrow} \mathrm{D}_{S}\right)=2
\end{array}
$$

Then, the relational forms associated to the above functions are defined as follows and depicted in Fig. 8

$$
\rho_{\text {target }_{\rightarrow}}=(\mathrm{X} \otimes \mathrm{I}) ;(\mathrm{I} \otimes \mathbf{X}) ;(\mathrm{V} \otimes \mathbf{I}) \quad \lambda_{\text {source }_{\rightarrow}}=(\mathrm{I} \otimes \Lambda) \quad \rho_{\text {lbl } \rightarrow}=\mathrm{I}_{3}
$$

Definition 6. Let $B=(Q, P, \rightarrow)$ be a component. The Petri Calculus term corresponding to the behaviour of $B$ in state $q \in Q$ is $\llbracket B \rrbracket: \underline{0} \rightarrow \underline{\# P}$, which is defined as follows

$$
T_{B_{q}} \stackrel{\text { def }}{=} d_{s} ;\left(\mathrm{I}_{s} \otimes\left(\rho_{\text {target }_{\rightarrow}} ; Q_{\{q\}} ; \lambda_{\text {source }_{\rightarrow}}\right)\right) ; \nabla_{s} ; \rho_{\text {lbl }} \rightarrow
$$

where

$$
Q_{Q^{\prime}} \stackrel{\text { def }}{=} \bigotimes_{i<\# Q} q_{i} \quad \text { where } \quad q_{i} \stackrel{\text { def }}{=} \begin{cases}\bullet & \text { if } i \in w_{Q}\left(Q^{\prime}\right) \\ \bigcirc & \text { otherwise }\end{cases}
$$




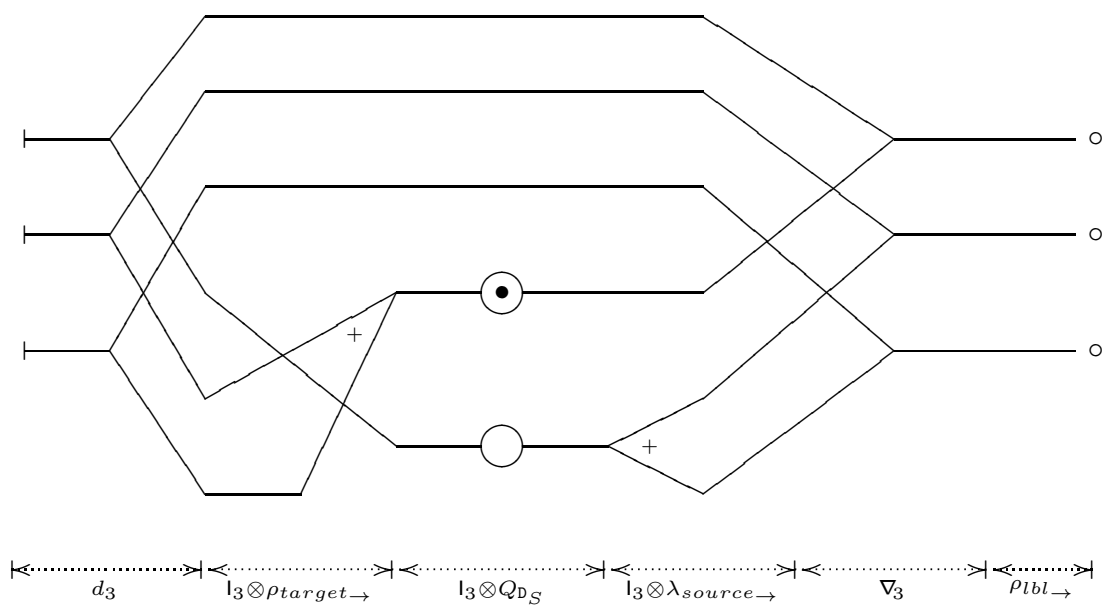

Fig. 9. Encoding of the $\mathrm{BI}(\mathrm{P})$ component Server

Example 4. Consider the component Server introduced in Example 1. Figure9 shows the term $T_{B_{\mathrm{D}_{S}}}$ corresponding to the encoding of the component Server for the initial state $\mathrm{D}_{S}$ and the ordering functions given in Example 3 .

The following results formalise the relation between the behaviour of components and their encodings. The first lemma is auxiliary and characterises the behavior of terms of the form $Q_{Q^{\prime}}$

Lemma 2. $Q_{Q^{\prime}} \stackrel{\ulcorner Z\urcorner}{\ulcorner W\urcorner} R$ iff $R=Q_{Q^{\prime \prime}}, W \subseteq Q^{\prime}, Z \cap Q^{\prime}=\varnothing$ and $Q^{\prime \prime}=\left(Q^{\prime} \backslash W\right) \cup$ $Z$.

Proof. Examination of either rules $\left(\perp_{1}\right)$ and $\left(\mathrm{T}_{1}\right)$, together with the rule (CuT) (when $p=0$ ) or rules (TкI) and (TкO), together with the rule (TeN) (when $p>0)$.

Next result ensures that the transitions of a component are in one-to-one correspondence with the moves of the corresponding Petri calculus term.

Theorem 1. Let $B=(Q, P, \rightarrow)$ a basic component. Then,

(i) if $q \stackrel{a}{\rightarrow} q^{\prime}$ then $T_{B_{q}} \underset{\left\ulcorner w_{P}(a)\right\urcorner}{\longrightarrow} T_{B_{q^{\prime}}}$.

(ii) if $T_{B_{q}} \underset{\beta}{\rightarrow} R$ then there exists $q^{\prime}$ s.t. $q \stackrel{a}{\rightarrow} q^{\prime}, R=T_{B_{q^{\prime}}}$ and $\beta=\left\ulcorner w_{P}(a)\right\urcorner$.

Proof. 1) By Lemma 2, $Q_{q} \underset{\left\ulcorner w_{Q}(q)\right\urcorner}{\stackrel{\left\ulcorner w_{Q}\left(q^{\prime}\right)\right\urcorner}{\ulcorner}} Q_{q^{\prime}}$. Then, by Lemma 1 and rule (CUT) after noting that source $\rightarrow_{\rightarrow}\left(w_{\rightarrow}\left(q \stackrel{a}{\rightarrow} q^{\prime}\right)\right)=w_{Q}(q)$ and $\operatorname{target}_{\rightarrow}\left(w_{\rightarrow}\left(q \stackrel{a}{\rightarrow} q^{\prime}\right)\right)=$ $w_{Q}\left(q^{\prime}\right)$, we have

$$
\left.\left.\left(\rho_{\text {target }_{\rightarrow}} ; Q_{\{q\}} ; \lambda_{\text {source }_{\rightarrow}}\right)\right) \underset{\left\ulcornerw _ { \rightarrow } \left( q^{\left.\left.\stackrel{a}{\rightarrow} q^{\prime}\right)\right\urcorner}\right.\right.}{\stackrel{\left.\left\ulcorner w_{\rightarrow} \stackrel{a}{\rightarrow} q^{\prime}\right)\right\urcorner}{\longrightarrow}}\left(\rho_{\text {target }_{\rightarrow}} ; Q_{\{q\}} ; \lambda_{\text {source }_{\rightarrow}}\right)\right)
$$


and subsequently by Proposition 1 and rules (CUT) and (TEN)

$$
\begin{array}{r}
d_{s} ;\left(\mathrm{I}_{s} \otimes\left(\rho_{\text {target } \rightarrow} ; Q_{\{q\}} ; \lambda_{\text {source }_{\rightarrow}}\right)\right) ; \nabla_{s} \stackrel{\left\ulcorner w_{\rightarrow}\left(q \stackrel{a}{\rightarrow} q^{\prime}\right)\right\urcorner}{\longrightarrow} \\
d_{s} ;\left(I_{s} \otimes\left(\rho_{\text {target }_{\rightarrow}} ; Q_{\{q\}} ; \lambda_{\text {source }_{\rightarrow}}\right)\right) ; \nabla_{s}
\end{array}
$$

The proof is completed by noting that $l b l_{\rightarrow}\left(w_{\rightarrow}\left(q \stackrel{a}{\rightarrow} q^{\prime}\right)\right)=w_{P}(a)$ and using Lemma 1 and rule (CUT) to conclude

$$
T_{B_{q}} \underset{\left\ulcorner w_{P}(a)\right\urcorner}{\longrightarrow} T_{B_{q^{\prime}}}
$$

2) If $T_{B_{q}} \underset{\beta}{\rightarrow} R$, then by rule (CUT) $\rho_{l b l_{\rightarrow}} \underset{\beta}{\stackrel{\alpha}{\rightarrow}} \rho_{l b l_{\rightarrow}}, \nabla_{s} ; \rho_{l b l} \rightarrow \underset{\beta}{\stackrel{\alpha \alpha}{\rightarrow}} \nabla_{s} ; \rho_{l b l \rightarrow}$ and

$$
\left(\mathrm{I}_{s} \otimes\left(\rho_{\text {target }_{\rightarrow}} ; Q_{\{q\}} ; \lambda_{\text {source }_{\rightarrow}}\right)\right) \underset{\alpha \alpha}{\stackrel{\alpha \alpha}{\alpha}}\left(\mathrm{I}_{s} \otimes R\right)
$$

Hence,

$$
\left(\rho_{\text {target }} \rightarrow Q_{\{q\}} ; \lambda_{\text {source }_{\rightarrow}}\right) \underset{\alpha}{\stackrel{\alpha}{\longrightarrow}} R
$$

The only non trivial transition implies $Q_{\{q\}} \underset{\ulcorner q\urcorner}{\stackrel{\left\ulcorner Q^{\prime}\right\urcorner}{\longrightarrow}} Q_{Q}^{\prime}$. By Lemma 1 and $w_{Q}(q)=$ source $\rightarrow\left(_{\rightarrow}^{\rightarrow}\left(q \stackrel{a}{\rightarrow} q^{\prime}\right)\right)$, we conclude

$$
\lambda_{\text {source }_{\rightarrow}} \stackrel{\left\ulcorner w_{Q}(q)\right\urcorner}{\alpha} \lambda_{\text {source }_{\rightarrow}}
$$

with $\alpha=\left\ulcorner w_{\rightarrow}\left(q \stackrel{a}{\rightarrow} q^{\prime}\right)\right\urcorner$. By reasoning analogously, on $\rho_{\text {target }_{\rightarrow}}$, we have that

$$
\rho_{\text {target }} \rightarrow \underset{\left\ulcorner w_{Q}\left(q^{\prime}\right)\right\urcorner}{\stackrel{\alpha}{\longrightarrow}} \rho_{\text {target }} \rightarrow
$$

Hence, $R=\left(\rho_{\text {target }_{\rightarrow}} ; Q_{\left\{q^{\prime}\right\}} ; \lambda_{\text {source }_{\rightarrow}}\right)$. The proof is completed by using Lemma 1 to conclude that $\rho_{l b l} \rightarrow \underset{\left\ulcorner w_{P}(a)\right\urcorner}{\stackrel{\alpha}{\longrightarrow}} \rho_{l b l_{\rightarrow}}$.

Example 5. It can be easily checked that the term $T_{B_{\mathrm{D}_{S}}}$ introduced in Example 4 has the following transitions:

$$
T_{B_{\mathrm{D}_{S}}} \underset{000}{\longrightarrow} T_{B_{\mathrm{D}_{S}}} \quad T_{B_{\mathrm{D}_{S}}} \underset{100}{\longrightarrow} T_{B_{\mathrm{C}_{S}}} \quad T_{B_{\mathrm{C}_{S}}} \underset{010}{\longrightarrow} T_{B_{\mathrm{D}_{S}}} \quad T_{B_{\mathrm{C}_{S}}} \underset{001}{\longrightarrow} T_{B_{\mathrm{C}_{S}}}
$$

that correspond to the transitions

$$
\mathrm{D}_{S} \stackrel{\varnothing}{\longrightarrow} \mathrm{D}_{S} \quad \mathrm{D}_{S} \stackrel{\text { acpt }}{\longrightarrow} \mathrm{C}_{S} \quad \mathrm{C}_{S} \stackrel{\text { ret }}{\longrightarrow} \mathrm{D}_{S} \quad \mathrm{C}_{S} \stackrel{\text { err }}{\longrightarrow} \mathrm{D}_{S}
$$

\subsection{Encoding of Interactions}

We now focus on the encoding of an interaction as a stateless connector. For $\alpha \in\{0,1\}^{h}$ a binary string of length $h>0$, we let $R^{\alpha}:(h, 1)$ denote the process inductively defined by:

$$
R^{0}=\downarrow ; \top \quad R^{1}=\mathrm{I} \quad R^{x \alpha}=\left(R^{x} \otimes R^{\alpha}\right) ; \nabla
$$

Intuitively, the term $R^{\alpha}$ synchronizes the ports associated to the positions of $\alpha$ that are set to 1 . 
Lemma 3. The process $R^{\alpha}$ is stateless for any $\alpha$, i.e., whenever $R^{\alpha} \underset{\beta^{\prime}}{\rightarrow} R^{\prime}$ then $R^{\prime}=R^{\alpha}$.

Proof. The thesis follows simply by noting that $R^{\alpha}$ is composed out of stateless connectors, i.e., the encoding does not exploit the constants $\bigcirc$, $\odot$.

Lemma 4. $R^{\alpha} \underset{\beta^{\prime}}{\stackrel{\beta}{\longrightarrow}} R^{\prime}$ iff

1. $\beta=0^{\# \alpha}$ and $\beta^{\prime}=0$; or

2. $\beta=\alpha$ and $\beta^{\prime}=1$.

Proof. The thesis follows by induction on the length of $\alpha$.

Definition 7. Let $\gamma$ be a finite set of interactions over a finite set of ports I, $j=\# I$ and $k=\# \gamma$. After fixing $w_{I}$ and $w_{\gamma}$, the encoding for the set $\gamma$ is

$$
\llbracket \gamma \rrbracket_{I}=\Lambda_{j}^{k} ;(\gamma)_{I} ; \Delta_{k} ;\left(\mathrm{I}_{k} \otimes\left(\mathrm{V}_{1}^{k} ; \perp\right)\right)
$$

where

$$
\begin{aligned}
\left(\{a\} D_{I}\right. & =R^{\left\ulcorner w_{I}(a)\right\urcorner} \\
\left(\{a\} \cup \gamma^{\prime} D_{I}\right. & =R^{\left\ulcorner w_{I}(a)\right\urcorner} \otimes\left(\gamma^{\prime} D_{I} \text { when } a \text { is the minimum in }\{a\} \cup \gamma^{\prime} \text { w.r.t. } w_{\gamma}\right.
\end{aligned}
$$

Example 6. Consider the set $\gamma=\{\{$ acpt, req $\},\{$ ret, resp $\},\{$ err, hdle $\}\}$ defined over $I=\{$ acpt, ret, err, req, resp, hdle $\}$ with the ordering functions defined as follows:

$$
\begin{aligned}
& w_{\gamma}(\{\text { acpt }, \text { req }\})=0 \quad w_{\gamma}(\{\text { ret, resp }\})=1 \quad w_{\gamma}(\{\text { err }, \text { hdle }\})=2 \\
& w_{I}(\text { acpt })=0 \quad w_{I}(\text { ret })=1 \quad w_{I}(\text { err })=2 \\
& w_{I}(\text { req })=3 \quad w_{I}(\text { resp })=4 \quad w_{I}(\text { hdle })=5
\end{aligned}
$$

Figure 6] shows the subterm $\Lambda_{6}^{3} ;(\gamma)_{I}$ (we use a compact representation in which a chain of several identical connectors like $\Lambda$ or $\nabla$ are collapsed in a unique node). Note that $(\gamma)_{I}=R^{\left\ulcorner w_{I}(\{\text { acpt,req }\})\right\urcorner} \otimes R^{\left\ulcorner w_{I}(\{\text { ret,resp }\})\right\urcorner} \otimes R^{\left\ulcorner w_{I}(\{\text { err, hdle }\})\right\urcorner}$ stands for the parallel evaluation of the three interactions in $\gamma$. The term $\Lambda_{6}^{3}$ ensures that conflicting interactions (i.e., the ones sharing a common action) are performed in mutual exclusion. We remark that the term $\Lambda_{6}^{3} ;(\gamma)_{I}$ still would allow for the concurrent execution of non-conflicting interactions (e.g., involving different sets of components). Since the semantics of $\mathrm{BI}(\mathrm{P})$ is purely sequential, we need to forbid the concurrent execution of disjoint interactions. This is ensured in the complete encoding by the subterm $\Delta_{3} ;\left(\mathrm{I}_{3} \otimes\left(\mathrm{V}_{1}^{3} ; \perp\right)\right)$.

The following results characterise the behaviour of $\llbracket \gamma \rrbracket_{I}$ and are instrumental to the proof of our main result (Theorem 2).

Lemma 5. Let $\gamma$ be a synchronization set over $I$ with $\# I=j$ and $\# \gamma=k$, and $w_{I}$ and $w_{\gamma}$ the functions sorting the elements of $I$ and $\gamma$.Then 


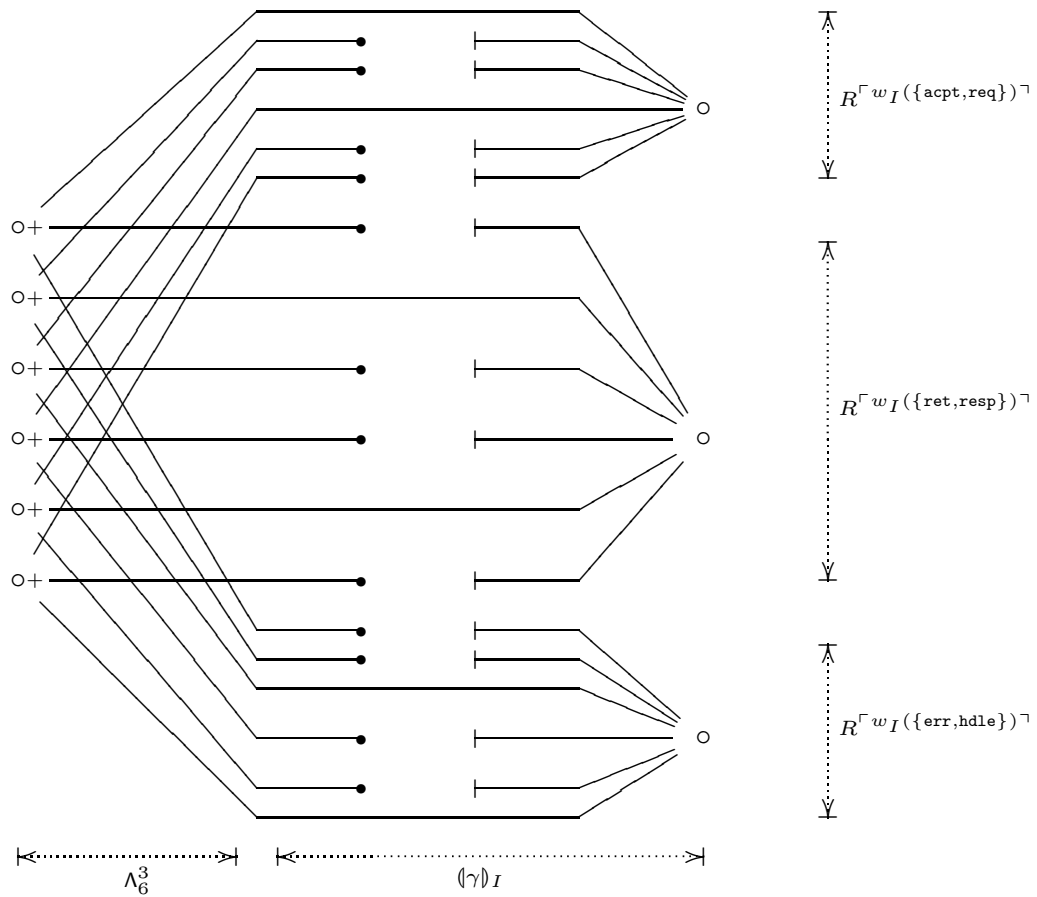

Fig. 10. Graphical representation of $\Lambda_{6}^{3} ;(\gamma)_{I}$

1. $(\gamma)_{I}:(j * k, k)$.

2. $(\gamma)_{I}$ is stateless for any $\gamma$, i.e., whenever $(\gamma)_{I} \underset{\beta}{\stackrel{\alpha}{\rightarrow}} R$ then $R=(\gamma)_{I}$.

3. $(\gamma)_{I} \underset{\beta}{\stackrel{\alpha}{\rightarrow}} R$ iff $\forall 0 \leq h \leq k-1$ either

$-\beta_{h}=0$ and $\forall j * h \leq i \leq j *(h+1)-1: \alpha_{i}=0$, or

$-\beta_{h}=1$, and $\exists a \in \gamma$ s.t. $w_{\gamma}(a)=h$ and $\alpha_{j * h . . j *(h+1)-1}=\left\ulcorner w_{I}(a)\right\urcorner$.

Proof. (1) Follows by induction on $\# \gamma$. (2) Follows by noting that $(\gamma)_{I}$ is composed out of stateless connectors, i.e., the encoding does not exploit the constants $\bigcirc, \odot$. (3) By induction on $h$ and using Lemma 4.

Lemma 6. Let $\gamma$ be a synchronization set over $I$ with $\# I=j$ and $\# \gamma=k$, and $w_{I}$ and $w_{\gamma}$ the functions sorting the elements of $I$ and $\gamma$.Then

1. $\llbracket \gamma \rrbracket_{I}:(j, k)$.

2. $\llbracket \gamma \rrbracket_{I}$ is stateless for any $\gamma$, i.e., whenever $\llbracket \gamma \rrbracket_{I} \underset{\beta}{\stackrel{\alpha}{\rightarrow}} R$ then $R=\llbracket \gamma \rrbracket_{I}$.

3. $\llbracket \gamma \rrbracket_{I} \underset{\beta}{\stackrel{\alpha}{\longrightarrow}} R$ iff $\exists a \subseteq \gamma$ s.t. $\# a \leq 1, \alpha=\left\ulcorner w_{I}(a)\right\urcorner$ and $\beta=\left\ulcorner w_{\gamma}(a)\right\urcorner$.

Proof. (1) Follows from the fact that: $\Lambda_{\# I}^{k}:(j, j * k) ;(\gamma)_{I}:(j * k, k)$ by Lemma 51); $\Delta_{k}:(k, 2 k)$; and $\left(\mathrm{I}_{k} \otimes\left(\mathrm{V}_{1}^{k} ; \perp\right)\right):(2 k, k)$. (2) Follows by noting that 
$(\gamma)_{I}$ is composed out of stateless connectors, i.e., the encoding does not exploit the constants $\bigcirc, \odot$. (3) By Proposition 10(5),

$$
\mathrm{V}_{1}^{k} \underset{\beta}{\stackrel{\alpha^{\prime}}{\rightarrow}} \mathrm{V}_{1}^{k}
$$

with $\# \alpha^{\prime}=k, \# \beta=1$ and $\beta=\sum_{j<k} \alpha_{j}^{\prime}$. Therefore, $\sum_{j<k} \alpha_{j}^{\prime} \leq 1$. By the semantics of $\perp$ and ; we have

$$
\mathrm{V}_{1}^{k} ; \perp \stackrel{\alpha^{\prime}}{\longrightarrow} \mathrm{V}_{1}^{k} ; \perp
$$

By using Proposition 1(2) and the inference rules for ; and $\otimes$

$$
\Delta_{k} ;\left(\mathrm{I}_{k} \otimes\left(\mathrm{V}_{1}^{k} ; \perp\right)\right) \underset{\alpha^{\prime}}{\stackrel{\alpha^{\prime}}{\longrightarrow}} \Delta_{k} ;\left(\mathrm{I}_{k} \otimes\left(\mathrm{V}_{1}^{k} ; \perp\right)\right)
$$

The proof is completed by using Lemma 6(3), Proposition(1) and the semantics of ;.

\subsection{Encoding of HBI(P) Systems}

The encoding of $\operatorname{HBI}(\mathrm{P})$ systems is defined by a suitable combination of the encoding of basic components and interactions.

Definition 8. Let $B$ be a $H B I(P)$ system with initial $q \in \mathbb{Q}_{B}$. The corresponding Petri Calculus term is inductively defined as follows.

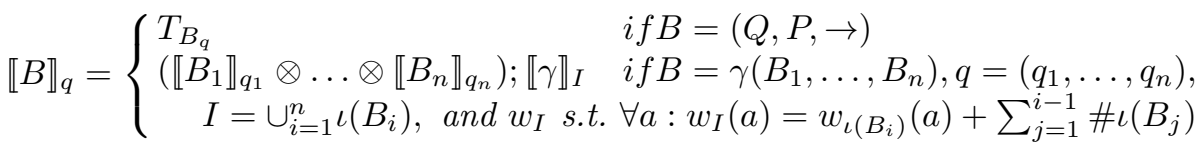

Theorem 2 (Correspondence). Let $B$ be a $H B I(P)$ system with initial state $q$. Then $q \stackrel{a}{\rightarrow} q^{\prime}$ if and only if $\llbracket B \rrbracket_{q} \underset{\left\ulcorner w_{\iota(B)(a)}\right\urcorner}{\longrightarrow} \llbracket B \rrbracket_{q^{\prime}}$.

Proof. By induction on the structure of the system $B$. Base case $(B=(Q, P, \rightarrow))$ follows by Theorem 1. Inductive step follows by applying inductive hypothesis on $\llbracket B_{1} \rrbracket_{q_{1}}, \ldots, \llbracket B_{n} \rrbracket_{q_{n}}$. Then, the proof is completed by using Lemma 6 .

\section{Conclusion}

This paper studies the hierarchical composition of $\mathrm{BI}(\mathrm{P})$ systems and its relation with the Petri calculus. For convenience of presentation we have chosen the particular variant of $\mathrm{BI}(\mathrm{P})$ consisting of the basic interaction model and purely sequential execution. Nevertheless, the results presented in this paper can be extended or adapted to handle several variants proposed in the literature. The remaining of this section is devoted to the discussion of some alternative presentations for $\mathrm{HBI}(\mathrm{P})$ and their relation with the encodings proposed in this paper. 
Concurrent executions. The work in [10] proposes a notion of hierarchical composition of $\mathrm{BI}(\mathrm{P})$ systems that allows for the concurrent execution of interactions, i.e., a set of conflict-free interactions (i.e., interactions that are pairwise disjoint) can be fired concurrently if enabled. We could encode such behaviour simply by defining $\llbracket \gamma \rrbracket_{I}$ as follows.

$$
\llbracket \gamma \rrbracket_{I}=\Lambda_{\# I}^{k} ;(\gamma)_{I}
$$

This definition simplifies Definition 7 by removing the subterm $\Delta_{k} ;\left(\mathrm{I}_{k} \otimes\left(\mathrm{V}_{1}^{k} ; \perp\right)\right)$. As already mentioned in $\S\left[4.2\right.$, the subterm $\left(\mathrm{V}_{1}^{k} ; \perp\right)$ ensures the execution of a unique interaction at a time. The results presented in the previous sections could also be formulated for this variant with minor adjustments.

For the sake of uniformity, the proposal in [10] also considers concurrent basic components instead of just sequential components as originally proposed in BIP. Concurrent basic components could be modelled as $\mathrm{C} / \mathrm{E}$ or $\mathrm{P} / \mathrm{T}$ nets with boundaries, which can be encoded as Petri calculus terms as shown in [9].

Triggers. In order to represent different modes of synchronisation, the BIP model has been extended with a sorting discipline for ports in 3. Typing associates synchronization types (trigger or synchron) to ports or connectors. The main difference is that an interaction $\left\{p_{1}, \ldots, p_{n}\right\}$ actually represents a set of interactions, i.e., all nonempty subset of $\left\{p_{1}, \ldots, p_{n}\right\}$ that contains some trigger; otherwise (if all of the ports are synchrons), the only possible interaction is the maximal one. Then, an interaction set $\gamma$ contains either standard interactions (i.e., without triggers), denoted as before by $a$, and connectors (i.e., interactions containing at least a trigger), denoted by c. Let c be a connector, we write $\gamma_{c}$ for the set of all standard denoted interactions (i.e., all subsets of $c$ that contains a trigger). By assuming a set of interactions, we extend Definition 7 with the rules for encoding connectors

$$
(\{c\})_{I}=\Lambda_{j}^{k} ;\left(\gamma \gamma_{c}\right)_{I} ; \mathrm{V}_{1}^{k}
$$

with $j=\# I$ and $k=\# \gamma_{\mathrm{c}}$ (rule for $\left(\{\mathrm{c}\} \cup \gamma^{\prime} D_{I}\right.$ is analogous).

By using Lemma 5 and the semantics of $\Lambda_{j}^{k}$ and $\bigvee_{j}^{k}$, it is easy to conclude that the only non trivial transitions of $\left(\{c \mathrm{c}\} D_{I}\right.$ are $\left(\{\mathrm{c}\} D_{I} \underset{1}{\stackrel{\alpha}{\rightarrow}}(\{\mathrm{c}\})_{I}\right.$ with $\alpha=\left\ulcorner w_{I}(a)\right\urcorner$ and $a \in \gamma_{\mathrm{c}}$. This characterization is analogous to the one for standard transitions in Lemma 4. This suffices to show that the correspondence results smoothly extend to the semantics of triggers.

Hiding. Hiding is an usual operator when composing systems hierarchically, because it enables components to compute internally. We can incorporate hiding to the definition of a $\operatorname{HBI}(\mathrm{P})$ system by adding the following item to Def. 5 .

- a composed system $B=\nu a B_{1}$ with interface $\iota(B)=\iota\left(B_{1}\right) \backslash\{a\}$ where $B_{1}$ is a hierarchical $\mathrm{BI}(\mathrm{P})$ system and $a \in \iota\left(B_{1}\right)$.

The semantics of $B=\nu a B_{1}$ is given by extending the definition of the space state of a $\operatorname{HBI}(\mathrm{P})$ system with the equation 
- $\mathbb{Q}_{B}=\left\{\nu a q \mid q \in \mathbb{Q}_{B_{1}}\right\}$ if $B=\nu a B_{1}$

and the following two inference rules

$$
\frac{q \stackrel{a}{\rightarrow} q^{\prime}}{\nu a q \stackrel{\varnothing}{\rightarrow} \nu a q^{\prime}} \quad \frac{a \neq b \quad q \stackrel{b}{\rightarrow} q^{\prime}}{\nu a q \stackrel{b}{\rightarrow} \nu a q^{\prime}}
$$

The encoding of HBI(P) system with hiding can be simply handled as follows

$$
\llbracket \nu a B \rrbracket_{\nu a q}=\llbracket B \rrbracket_{q} ; H^{\left\ulcorner w_{\iota(B)}(\{a\})\right\urcorner}
$$

where $H^{\alpha}$ for $\alpha \in\{0,1\}^{h}$ is the the process inductively defined by:

$$
H^{0}=\mathrm{I} \quad H^{1}=\perp \quad H^{x \alpha}=\left(H^{x} \otimes H^{\alpha}\right)
$$

We remark that the term $H^{\alpha}$ replicates on the right interface only the ports of the left interface that are in the positions of $\alpha$ and that are set to 0 , while the others are kept hidden. Consequently, $\left.H^{\left\ulcorner w_{\iota(B)}\right.}(\{a\})\right\urcorner$ hides the port associated to $a$. The extension of the correspondence results to $\mathrm{HBI}(\mathrm{P})$ systems with hiding is straightforward.

\section{References}

1. Arbab, F., Bruni, R., Clarke, D., Lanese, I., Montanari, U.: Tiles for Reo. In: Corradini, A., Montanari, U. (eds.) WADT 2008. LNCS, vol. 5486, pp. 37-55. Springer, Heidelberg (2009)

2. Basu, A., Bozga, M., Sifakis, J.: Modeling heterogeneous real-time components in BIP. In: Fourth IEEE International Conference on Software Engineering and Formal Methods (SEFM 2006), pp. 3-12. IEEE Computer Society (2006)

3. Bliudze, S., Sifakis, J.: The algebra of connectors - structuring interaction in BIP. IEEE Trans. Computers 57(10), 1315-1330 (2008)

4. Bliudze, S., Sifakis, J.: Causal semantics for the algebra of connectors. Formal Methods in System Design 36(2), 167-194 (2010)

5. Bruni, R., Lanese, I., Montanari, U.: A basic algebra of stateless connectors. Theor. Comput. Sci. 366(1-2), 98-120 (2006)

6. Bruni, R., Melgratti, H., Montanari, U.: A connector algebra for P/T nets interactions. In: Katoen, J.-P., König, B. (eds.) CONCUR 2011. LNCS, vol. 6901, pp. 312-326. Springer, Heidelberg (2011)

7. Bruni, R., Melgratti, H., Montanari, U.: Connector algebras, Petri nets, and BIP. In: Clarke, E., Virbitskaite, I., Voronkov, A. (eds.) PSI 2011. LNCS, vol. 7162, pp. 19-38. Springer, Heidelberg (2012)

8. Bruni, R., Melgratti, H., Montanari, U.: A survey on basic connectors and buffers. In: Beckert, B., Damiani, F., de Boer, F.S., Bonsangue, M.M. (eds.) FMCO 2011. LNCS, vol. 7542, pp. 49-68. Springer, Heidelberg (2012)

9. Bruni, R., Melgratti, H.C., Montanari, U., Sobocinski, P.: Connector algebras for C/E and P/T nets' interactions. Logical Methods in Computer Science 9(3) (2013)

10. Graf, S., Quinton, S.: Contracts for BIP: Hierarchical interaction models for compositional verification. In: Derrick, J., Vain, J. (eds.) FORTE 2007. LNCS, vol. 4574, pp. 1-18. Springer, Heidelberg (2007)

11. Sobociński, P.: Representations of Petri net interactions. In: Gastin, P., Laroussinie, F. (eds.) CONCUR 2010. LNCS, vol. 6269, pp. 554-568. Springer, Heidelberg (2010) 